\title{
Las perspectivas de la democracia sindical en Italia: el referéndum de los trabajadores
}

\author{
Davide Carbonai \\ Université Catholique de Louvain-la-Neuve (UCL), Lovaina, Bélgica. \\ Email: davide.carbonai@uclouvain.be
}

\begin{abstract}
Resumen: El objetivo de este estudio es destacar las nuevas formas de participación social en Italia. En primer lugar, se debatirán las principales características del referéndum de los trabajadores de octubre del 2007. Después, serán separados los datos por sexo, edad, y sector económico. Este estudio inicial nos dará las herramientas para el análisis de características y tendencias de la democracia sindical y cultura política Italiana. Para concluir, se propone una interpretación de la participación política en Italia.
\end{abstract} política.

Palabras clave: Democracia sindical; Italia; acción sindical; cultura

\section{The prospects of Union democracy in Italy: workers referendum}

\begin{abstract}
The aim of this paper is to point out recent union tendencies and forms of social participation in Italy. Preliminary, it debates the main features of the workers' referendum on October 2007. Secondly, disaggregating data by gender, age, economic sector, this study will provide tools for analysing general tendencies and features in union democracy and Italian political culture. In the conclusion, a general interpretation on political participation in Italy is proposed.

Key words: Union democracy; Italy; labor union action; political culture.
\end{abstract}

\section{As perspectivas para a democracia sindical na Itália: o referendo dos trabalhadores}

Resumo: O objetivo deste estudo é destacar as novas formas de participação social na Itália. Em primeiro lugar, irá discutir as principais características do referendo dos trabalhadores em outubro de 2007. Em seguida, os dados serão separados por sexo, idade e setor econômico. Este estudo inicial nos dará as ferramentas para analisar padrões e tendências da democracia sindical ea cultura política da Itália. Em conclusão, propomos uma interpretação da participação política na Itália. lítica.

Palavras-chave: Democracia sindical; Itália; atividade sindical, cultura po- 
En julio del 2007, en concierto con la política social, el gobierno Prodi y los principales sindicatos italianos (Cgil, Cisl y Uil) firman un acuerdo (Protocolo sobre el bienestar, el trabajo y la competitividad por la igualdad y el crecimiento sostenible, del 23 de julio de 2007. En octubre, los tres sindicatos Cgil, Cisl y Uil promueven un amplio referéndum para la aceptación o el rechazo del acuerdo realizado con el Gobierno Prodi. Más del 80 por ciento vota a favor del acuerdo entre el gobierno y sus interlocutores sociales, pero lo que llamó más la atención fue el alto grado de participación en el referéndum: más de cinco millones de votantes (Carrieri 2008: 10). Además, la complejidad de los temas tratados - política de providencia social, política del trabajo, y política de seguridad social - podría haber perjudicado dicha participación y el consenso de la misma (Accornero 2007).

En relación al caso italiano, esta unilateralidad sindical contrasta con un mundo del trabajo dividido y segmentado a varios niveles. Muchas encuestas, por ejemplo, confirman condiciones de desigualdad y pobreza (Ricolfi 2005; Istat 2008). Italia es uno de los países de la Unión Europea con mayor desigualdad de renta per capita: el valor de concentración de Gini está por encima de la media UE (Carbonai et al. 2009).

Esta fragmentación social se observa a varios niveles. Una vez más, las regiones italianas del Sur se caracterizan hoy por el aumento de las situaciones de sufrimiento social: privación, y pobreza, que se alargan por todos los grupos profesionales. Existen algunas diferencias, como la de los trabajadores cualificados y aquéllos que trabajan en profesiones técnicas, intelectuales o científicas; en cualquier caso, las diferencias entre las regiones del sur y las del norte son reducidas (Paci 2007).

La desigualdad por género es característica del mercado laboral italiano. El 48,9\% de las mujeres tiene una ganancia de hasta 1.000 euros al mes (contra 26,8\% de los hombres), y apenas 9\% llega alrededor de los 1.500 euros (contra 21,5\% de los hombres); una desventaja observada en todos los cargos profesionales, y a lo largo de las escalas educativa y profesional (Altieri et al. 2007).

El sistema del trabajo está fragmentado también con las diferencias existentes entre los trabajadores con contrato permanente y los temporarios. En pocas palabras, y por señalar un posterior elemento de división en el mundo del trabajo italiano, el 67\% gana menos de 1000 euros al mes y el $37,7 \%$ no exceden los 800 euros (versus respectivamente $24 \%$ y $7,4 \%$ de aquellos que son reclutados con contracto fijo) (Regalia 2005; Altieri et al. 2007).

Frente a estas características sociales y económicas - y alineada con una política de concertación social - el "Protocolo por el Bienestar Social" de Julio del 2007 intenta una respuesta política de concertación social a esta difícil situación. 
En la primera parte de este estudio — con base en los datos oficiales entregados por los sindicatos - son examinadas las principales estadísticas sobre la participación y votación según datos territoriales (el conjunto de datos está organizado sobre la base de la votación en cada provincia). En el segundo, se analizan los datos de una encuesta — una muestra de 1574 personas entrevistadas - con el fin de detectar algunos elementos característicos de la población en relación con el comportamiento de voto. En la última parte, se ofrece un panorama general y ciertas perspectivas para el sindicalismo italiano.

\section{Estadísticas del voto}

El referéndum de octubre de 2007 marca una mayor participación que en el anterior de 1995 (la reforma del sistema de jubilación propuesta por el gobierno Dini): 5.128.507 votos emitidos en 2007 contra 3.786.586 del referéndum de 1995. Por lo tanto, es un diferencial con una clara señal positiva: hubo 1.341.921 votantes más que en la consulta de 1995.

Teniendo en cuenta la población residente con más de quince años de edad, un promedio de 8,3\% de la población ha expresado una intención de voto. La participación por área geográfica indica un elemento de discontinuidad en relación al referéndum de 1995. Las cifras del 2007 marcan, en particular, un aumento del total de votantes en las regiones del sur: Sicilia, Campania, Apulia y el Lazio. La participación popular en Sicilia por ejemplo, crece en 462.598 unidades de votos emitidos. Se viene de este modo a modificar el peso de cada región sobre los datos agregados de la afluencia (en otras palabras, la participación sindical). Y del mismo modo, el peso de las macro-reparticiones territoriales; el Sur expresa el 19,64\% del total de los votos en 1995, el 35\% en 2007. En cambio, los votos emitidos por las regiones centrales y del Norte pesan menos: en las regiones centrales se pasa del 35,55\% en 1995 al 29,55\% del total de los votos en 2007; en el Norte, del 44,82\% en 1995 al 35,42\% en 2007.

Los resultados de la consulta de octubre de 2007 ofrecen, en su totalidad, una imagen clara de las elecciones de voto: de un total de 5.128.507 votantes, los votos a favor del acuerdo son iguales a $81,61 \%$ del total, en comparación con el 18,39\% de los contrarios ${ }^{1}$. Las regiones de Sicilia, Apulia, Valle de Aosta, Calabria, Campania y Cerdeña expresan el más alto porcentaje de votos favorables al acuerdo; en Lombardía, Piemonte y en el Abruzzo, las más bajas — donde uno de cada cuatro votantes expresa un voto contrario. Solo siete provincias del total de las unidades de análisis - expresan niveles de consenso inferiores al 70 por ciento.

El porcentaje de favorables y su variabilidad (en la distribución por categoría contractual), ofrece una primera fácil interpretación de los datos también por medio de una clasificación en cuatro grandes grupos de traba- 
jadores. En este sentido, en relación con el conjunto de datos, el primer grupo está integrado por los jubilados (un muy alto grado de consenso y baja variabilidad de los porcentajes de votos por provincia), el segundo está integrado por los metalúrgicos (bajo grado de consenso y alta variabilidad), la tercera de los atípicos (alto grado de consenso y altísima variabilidad) y el cuarto está compuesto por todos los demás contratos (baja variabilidad, alto grado de consenso). En otras palabras el voto de los metalúrgicos y de los atípicos - trabajadores temporarios - se diferencian del resto de la población de los trabajadores. Es importante recordar que el sindicato de categoría Fiom-Cgil (el sindicatos de los metalúrgicos) se revela en contra del acuerdo.

Los datos oficiales entregan otra importante información; sin embargo, cabe señalar un hecho: el número de votos por provincia —y por ende el valor absoluto - marca una plena dependencia hacia el número de afiliaciones sindicales (el índice de correlación de Pearson es igual a 0,92). El número de los inscritos a las organizaciones sindicales por cada provincia se correlaciona con el número de votos por provincia, por lo que, al aumentar el número de inscritos aumentarían linealmente los votos por provincia (Feltrin 2005): mientras más miembros, más votos. Por lo tanto se trata de un amplio consenso, pero limitado por la pertenencia sindical ${ }^{2}$.

\section{Análisis de la encuesta}

Además de los datos oficiales, aquí se presentan los perfiles sociales de la votación, derivados del análisis de un segundo conjunto de datos; la encuesta —realizada a partir de abril de 2008 sobre una muestra nacional, estratificada por cuotas, de 1574 sujetos- está dirigida a la comprensión de las relaciones entre la participación, la elección de voto y la cultura sindical, incluso las otras variables como el género, los diferentes grupos de edad y la profesión.

La muestra es representativa de la población italiana superior a los dieciocho años de edad: los empleados en posiciones subordinadas - tanto en el sector privado de la economía como en el sector público- o jubilados. La muestra está formada por cuotas sacadas de las listas de los nominativos reportados en los elencos telefónicos de la entera red nacional. Los métodos utilizados para identificar las unidades finales son de tipo aleatorio estratificado en cuatro niveles: sexo, zona geográfica, clase de la amplitud demográfica del municipio, condición profesional. Los cuatro parámetros son uniformados por los datos proporcionados por el ISTAT (según el Censo General de Población y el Anuario de Estadística Italiano), identificadas las «muestras-comunes» se inició la recolección de datos. El estudio fue realizado utilizando un cuestionario estructurado aplicado tanto por vía telefónica (sistema CATI - Computer Assisted Telephone Interview) como online (CAWI - Computer Assisted Web Interview). 


\section{Cuadro 1 - Muestra de la población: sexo y distribución geográfica (en porcentaje)}

\begin{tabular}{|l|c|c|c|}
\hline Condición de empleo & Hombres & Mujeres & Total \\
\hline Empleados & 34,7 & 25,8 & 60,5 \\
\hline Jubilados & 22,1 & 17,4 & 39,5 \\
\hline Total & 56,8 & 43,2 & 100 \\
\hline \multicolumn{3}{|l|}{} \\
\hline Área geográfica & Hombres & Mujeres & Total \\
\hline Noroeste & 17,0 & 12,9 & 29,9 \\
\hline Noreste & 12,5 & 10,2 & 22,6 \\
\hline Centro & 10,7 & 9,0 & 19,7 \\
\hline Sur & 10,9 & 6,1 & 17,0 \\
\hline Islas & 6,5 & 4,3 & 10,8 \\
\hline Total & 57,5 & 42,5 & 100 \\
\hline
\end{tabular}

La población, distinta según cohortes de edad, se distribuye en seis clases de diferentes dimensiones. La clase 55-64 años (26,2\%) es la más grande; sobre los 64 años tenemos el 16,6\%; por debajo de 44 años se incluye el $42 \%$ de la población (el 19,63\% en la cohorte de 35 a 44 años, el $20,2 \%$ en la cohorte entre 25 y 34 años, y el 1,91\% para los jóvenes de edades comprendidas entre los 18 y 24 años). Se puede considerar que en la muestra de población no se incluyen estudiantes, amas de casa y otras categorías de no-trabajadores.

El 62\% de los encuestados son empleados —o al menos lo era antes de la jubilación- en el sector privado de la economía (y el resto en el sector público). Se trata de una población compuesta mayormente de empleados genéricos (o ex-empleados), de concepto o cuadros dirigentes.

Se subraya el interés de los encuestados - y no sólo de los votantes - en relación al contenido del acuerdo. El 53,7\% de los encuestados afirman conocer el contenido ${ }^{3}$. Por lo tanto se trata de una parte -informada sobre el contenido del acuerdo- mucho más importante que los simples votantes (el 33,9\% de la muestra). Distinguida por género, la distribución de los «informados» pesa en favor de los hombres: que son «muy informados o informados» el 62,2\% de los varones frente a $41 \%$ de las mujeres. Entre los 18 y 24 años sólo el 40\% está «informado» o «muy informado». La estadística asciende en las cohortes de edad superiores: el $43 \%$ entre 25 y 34 años, el 47\% entre 35 y 44, el 61,2\% entre 45 y 54 años, hasta un máximo nivel de entre 55 y 64 años $(62,4 \%)^{4}$.

Además que por cuestiones de género y edad, considerando sólo la población activa, la distribución de los «informados» se asocia con el tipo de contrato de trabajo. A ser «informado» o «muy informado» es el 54,5\% de los empleados con contrato a tiempo indeterminado contra el 38,3\% de los empleados con formas de trabajo flexible.

La inscripción a un sindicato es un factor importante en la compren- 
sión de las estadísticas de participación. Las diferencias más marcadas se observan entre los «miembros» o «los que piensan en inscribirse» y los que, en cambio, asumen una actitud de «negación/oposición» hacia las organizaciones sindicales («No, no deseo inscribirme»). Alrededor de la mitad de los miembros deciden de participar en la votación (y el $48 \%$ entre quienes no están inscritos, pero está «pensando inscribirse»). Este porcentaje se reduce al $23,97 \%$ entre trabajadores que actualmente no son miembros y no tienen intención de inscribirse en el futuro. La capacidad de penetración social del sindicato aparece delimitada por los confines sociales - muy vastos - de la pertenencia sindical.

\section{Cuadro 2 - Tabla de contingencia: afiliación a un sindicato y propensión a votar}

\begin{tabular}{|l|l|c|c|c|}
\hline \multirow{2}{*}{\multicolumn{2}{|c|}{$\mathrm{N}=1574$}} & \multicolumn{2}{|c|}{$\begin{array}{c}\text { Fue a votar a este } \\
\text { referéndum? }\end{array}$} & \multirow{2}{*}{ Total } \\
\cline { 3 - 5 } & & $\mathrm{Si}$ & \multirow{2}{*}{ No } & \\
\hline \multirow{4}{*}{$\begin{array}{l}\text { ¿Actualmente, está } \\
\text { inscrito en un } \\
\text { sindicato? }\end{array}$} & $\mathrm{Sí}$ & $50,22 \%^{*}$ & $49,78 \% \%^{* *}$ & 100 \\
\cline { 2 - 5 } & No, pero creo que me inscribiré & $48,96 \% *$ & $51,04 \%$ & 100 \\
\cline { 2 - 5 } & No, pero yo estaba escrito en el & $27,96 \% * *$ & $72,04 \%$ & 100 \\
\cline { 2 - 5 } & No, yo no deseo inscribirme & $23,97 \%^{* *}$ & $76,03 \% *$ & 100 \\
\cline { 2 - 5 } & Prefiero no responder & $24,49 \%$ & $75,51 \%$ & 100 \\
\cline { 2 - 5 } & Total & $33,93 \%$ & $66,07 \%$ & 100 \\
\hline
\end{tabular}

*Standard residual superior a 2 (fuerte asociación positiva)

** Standard residual inferior a -2 (fuerte asociación negativa)

Aunque menos informados, la propensión de voto de los empleados con tipologías de contrato atípicos resulta casi alineada con la de los empleados con contratos permanentes: el $41,5 \%$ de los empleados permanentes fue a votar, contra el $37,4 \%$ de los trabajadores discontinuos (con un valor medio de 40,8\%, calculado sobre el total de los ocupados).

En cuanto al sector económico — considerando sólo la parte de los ocupados - sólo en la industria metalúrgica se observan estadísticas de participación significativamente superiores a la media de la muestra de población: el $56,1 \%$ de los trabajadores del sector metalúrgico decide votar, en comparación con el 40,3\%, calculado como media de la población ocupada.

La participación de los trabajadores resulta ser superior a la media (con una diferente propensión entre obreros especializados y obreros no especializados). El porcentaje de votantes entre los profesores es significativamente inferior a los valores medios. Inferior a la media es la participación en general de los empleados genéricos, y superior la de los empleados directivos.

Además, la tabla de contingencia nos informa de una independencia entre el «componente política» y la "participación en las votaciones” si no 
en correspondencia de la modalidad «centro-derecha» (componente política en apoyo de Silvio Berlusconi) donde vota el 27,33\% frente a una media del 33,9\% (se trata por tanto de una asociación negativa) ${ }^{5}$.

El 66,1\% de la muestra de población no va a las urnas. Las razones para no votar se relacionan en su mayoría con la insuficiente información sobre el contenido (19,4\%) o el total desinterés ( «no me interesa», 17,8\%). Una gran proporción de no-votantes se compone de aquellos que no votaron porque no estaban informados de la realización de referéndum (12,9\%).

Teniendo en cuenta sólo la parte de los votantes — 534 encuestadosel 54,3\% de ellos declara haber votado a favor de la cuestión del referéndum - y por tanto a favor del Acuerdo de julio - contra el 26,4\% de los contrarios, el $4,9 \%$ de los votantes con tarjetas en blancos (o nulas) y el $14,4 \%$ que prefiere no responder ${ }^{6}$.

En cambio, la pertenencia a un sindicato marca una distinción respecto a la media analizada. Teniendo en cuenta la población de los votantes, el 68,89\% de los afiliados en alguna organización sindical ejerce el voto a favor del acuerdo. Entre aquellos que desearan unirse - «No, pero estoy pensando en inscribirme» - la propensión a votar resulta menor equivalente al 57,45\% - y de todas maneras superior que la media de la población. Entre la población de los «decepcionados» («No, pero estaba inscrito en el pasado») la propensión baja al 48,08\%.

\section{Cuadro 3 - Votación y afiliarse a un sindicato (porcentajes)}

\begin{tabular}{|c|c|c|c|c|c|c|}
\hline & \multirow{2}{*}{$\mathrm{N}=534$} & \multicolumn{4}{|c|}{$\begin{array}{c}\text { ¿Ha votado a favor o contra } \\
\text { el Protocolo? }\end{array}$} & \multirow{2}{*}{ Total } \\
\hline & & $\begin{array}{c}\text { A } \\
\text { Favor }\end{array}$ & Contra & $\begin{array}{l}\text { He votado en } \\
\text { blanco - } \\
\text { Votos nulos }\end{array}$ & $\begin{array}{c}\text { Prefiero no } \\
\text { responder }\end{array}$ & \\
\hline \multirow{5}{*}{$\begin{array}{l}\text { ¿Actualmente, } \\
\text { está inscrito } \\
\text { en un } \\
\text { sindicato? }\end{array}$} & Sí & $68,89^{*}$ & 22,67 & $1,33^{* *}$ & $7,11^{* *}$ & 100 \\
\hline & $\begin{array}{l}\text { No, pero creo que me } \\
\text { inscribiré }\end{array}$ & 48,08 & $35,58^{*}$ & 2,88 & 13,46 & 100 \\
\hline & $\begin{array}{l}\text { No, pero yo estaba } \\
\text { inscrito en el pasado }\end{array}$ & 57,45 & 19,15 & 6,38 & 17,02 & 100 \\
\hline & \begin{tabular}{|l|} 
No, yo no deseo \\
inscribirme
\end{tabular} & $36,99^{* *}$ & 29,45 & 10,96 & 22,60 & 100 \\
\hline & Prefiero no responder & 33,33 & 8,33 & 8,33 & 50,00 & 100 \\
\hline \multicolumn{2}{|l|}{ Total } & 54,31 & 26,40 & 4,87 & 14,42 & 100 \\
\hline
\end{tabular}

*Standard residual superior a 2 (fuerte asociación positiva)

** Standard residual inferior a -2 (fuerte asociación negativa)

Teniendo en cuenta la población de votantes, el 71\% que «políticamente se colocaría con mayor facilidad» en el segmento de «centro-izquier- 
da» opta por votar a favor del acuerdo. Entre los votantes-electores de «centro-derecha» este porcentaje se reduce al $40 \%$. Significativo es el $40,8 \%$ de aquellos que se colocan a «izquierda» y que deciden votar en contra del acuerdo.

La votación a favor del acuerdo se distribuye casi por igual entre los diversos grupos profesionales, con picos entre los obreros cualificados (61,5\%) y los cuadros directivos (58,16\% a favor del acuerdo).

\section{Confianza institucional}

Basándose en el tipo de voto es posible obtener una tipología de cuatro actitudes relativas a lo Referéndum de Octubre: favorables, freeriders (no participan en la votación pero son favorables), contrarios, indiferentes.

\section{Cuadro 4 - Actitud de voto sobre el referéndum}

\begin{tabular}{|l|l|c|c|l|}
\hline & Tipo & $\begin{array}{l}\text { Número de } \\
\text { casos }\end{array}$ & Porcentaje & Caracteristicas del tipo \\
\hline 1 & Favorables & 290 & 19,72 & Participan en la votación y votan a favor \\
\hline 2 & Free-riders & 318 & 21,62 & No participan en la votación pero son favorables \\
\hline 3 & Contrarios & 377 & 25,63 & $\begin{array}{l}141 \text { participan en la votación y votan contra } \\
(9,5 \%) ; 236 \text { no votan pero son contra (16,04\%) }\end{array}$ \\
\hline 4 & Indiferentes & 486 & 33,03 & $\begin{array}{l}\text { No votan y no tienen puntos de vista sobre del } \\
\text { Acuerdo }\end{array}$ \\
\hline \multicolumn{2}{|l|}{ Total } & 1471 & 100 & \\
\hline
\end{tabular}

Incluidos entre los favorables son especialmente los trabajadores especializados (33\% versus el 19,72\% como valor calculado sobre la muestra). Particularmente marcada la propensión de los miembros el sindicato (36\% de ellos) y de aquellos que quisieran unirse a un sindicato (32\%) que han de incluirse entre los favorables. Además, se trata fundamentalmente de los votantes de centro-izquierda quienes aparecen entre los favorables (27\%).

Entre las mujeres se reduce la proporción de las contrarias (no-votantes); el 11\% contra el 16\%, calculado sobre la muestra. Entre los contrarios no-votantes aparecen principalmente ejecutivos y académicos.

Mientras entre los hombres el porcentaje de los indiferentes se sitúa en el $24,64 \%$, entre las mujeres el porcentaje se eleva a 46,32\%. Aparecen sobretodo los empleados en esta clase, el $42 \%$ entre los genéricos en frente a una media del 33,03\%. 
También el contexto institucional — tal como percibido por el entrevistado- sigue siendo asociado con el comportamiento de voto. En particular, el cuestionario tiene un número de ítems — una batería de preguntas en escala Likert 1-5- diseñado para detectar los niveles de confianza hacia siete instituciones (parlamento, gobierno, partidos políticos, asociaciones empresariales, sindicatos, magistratura, Iglesia Católica).

Lo que llama la atención es el nivel general de confianza muy bajo: que indica una crisis de las instituciones italianas. El Poder Judicial $(3,01)$ es la institución con mayor puntuación de confianza percibida. Los partidos políticos $(2,06)$ y la asociación de empresarios $(2,24)$ son las categorías con las puntuaciones promedio de confianza más bajos.

\section{Cuadro 5 - Confianza en las instituciones y aptitud sobre el referéndum}

\begin{tabular}{|c|c|c|c|c|c|}
\hline Instituciones & \begin{tabular}{|l|l|} 
Tipología: \\
aptitud de voto sobre el \\
referéndum
\end{tabular} & $\mathrm{N}$ & Valor medio & $\mathrm{F}$ & Sig. \\
\hline \multirow{5}{*}{ Parlamento } & \begin{tabular}{|l|} 
Favorables \\
\end{tabular} & 287 & 2,80 & \multirow{5}{*}{11,151} & \multirow{5}{*}{, 000} \\
\hline & \begin{tabular}{|l} 
Free-riders \\
\end{tabular} & 314 & 2,76 & & \\
\hline & \begin{tabular}{|l|} 
Contrarios \\
\end{tabular} & 376 & 2,42 & & \\
\hline & \begin{tabular}{|l|} 
Indecisos \\
\end{tabular} & 451 & 2,45 & & \\
\hline & \begin{tabular}{|l|} 
Total \\
\end{tabular} & 1428 & 2,58 & & \\
\hline \multirow{5}{*}{ Gobierno } & Favorables & 285 & 2,44 & \multirow{5}{*}{1,136} & \multirow{5}{*}{, 333} \\
\hline & \begin{tabular}{|l|} 
Free-riders \\
\end{tabular} & 313 & 2,32 & & \\
\hline & \begin{tabular}{|l|} 
Contrarios \\
\end{tabular} & 373 & 2,28 & & \\
\hline & \begin{tabular}{|l|} 
Indecisos \\
\end{tabular} & 451 & 2,31 & & \\
\hline & Total & 1422 & 2,33 & & \\
\hline \multirow{5}{*}{ Partidos Políticos } & Favorables & 289 & 2,46 & \multirow{5}{*}{27,639} & \multirow{5}{*}{, 000} \\
\hline & \begin{tabular}{|l|} 
Free-riders \\
\end{tabular} & 314 & 2,18 & & \\
\hline & \begin{tabular}{|l|} 
Contrarios \\
\end{tabular} & 376 & 1,90 & & \\
\hline & Indecisos & 459 & 1,86 & & \\
\hline & \begin{tabular}{|l|} 
Total \\
\end{tabular} & 1438 & 2,06 & & \\
\hline \multirow{5}{*}{$\begin{array}{l}\text { Asociaciones } \\
\text { empresariales }\end{array}$} & Favorables & 288 & 2,33 & \multirow{5}{*}{1,141} & \multirow{5}{*}{, 331} \\
\hline & \begin{tabular}{|l|} 
Free-riders \\
\end{tabular} & 314 & 2,23 & & \\
\hline & \begin{tabular}{|l|} 
Contrarios \\
\end{tabular} & 373 & 2,18 & & \\
\hline & \begin{tabular}{|l} 
Indecisos \\
\end{tabular} & 455 & 2,24 & & \\
\hline & \begin{tabular}{|l|} 
Total \\
\end{tabular} & 1430 & 2,24 & & \\
\hline \multirow{5}{*}{ Sindicatos } & Favorables & 288 & 2,95 & \multirow{5}{*}{70,269} & \multirow{5}{*}{, 000} \\
\hline & \begin{tabular}{|l|} 
Free-riders \\
\end{tabular} & 315 & 2,55 & & \\
\hline & \begin{tabular}{|l|} 
Contrarios \\
\end{tabular} & 376 & 1,94 & & \\
\hline & \begin{tabular}{|l|} 
Indecisos \\
\end{tabular} & 462 & 2,08 & & \\
\hline & \begin{tabular}{|l|} 
Total \\
\end{tabular} & 1441 & 2,32 & & \\
\hline \multirow{5}{*}{ Magistratura } & Favorables & 288 & 3,33 & \multirow{5}{*}{19,781} & \multirow{5}{*}{, 000} \\
\hline & \begin{tabular}{|l} 
Free-riders \\
\end{tabular} & 317 & 3,29 & & \\
\hline & \begin{tabular}{|l|} 
Contrarios \\
\end{tabular} & 375 & 2,75 & & \\
\hline & \begin{tabular}{|l} 
Indecisos \\
\end{tabular} & 472 & 2,83 & & \\
\hline & \begin{tabular}{|l|} 
Total \\
\end{tabular} & 1452 & 3,01 & & \\
\hline \multirow{5}{*}{ Iglesia Católica } & Favorables & 289 & 2,54 & \multirow{5}{*}{1,553} & \multirow{5}{*}{, 199 } \\
\hline & \begin{tabular}{|l|} 
Free-riders \\
\end{tabular} & 316 & 2,48 & & \\
\hline & \begin{tabular}{|l|} 
Contrarios \\
\end{tabular} & 374 & 2,66 & & \\
\hline & \begin{tabular}{|l|} 
Indecisos \\
\end{tabular} & 462 & 2,48 & & \\
\hline & otal & 1441 & 2,54 & & \\
\hline
\end{tabular}

Nota (Pregunta 59): En una escala de 1 a 5, donde 1 = Ninguna e 5 = Mucha, ¿cuánta confianza tiene en la siguiente institución? 
Las puntuaciones medias del nivel de confianza pueden ser relacionadas con los distintos tipos (favorables, free-riders, contrarios e indiferentes). Tendencialmente los valores de la confianza institucional son superior entre los favorables, inferior entre los free-riders, aún más bajos entre los contrarios y los indiferentes. El contexto institucional - tal como percibido - se asocia con el comportamiento de voto ${ }^{7}$.

No son especialmente altas las puntuaciones atribuidas a los sindicatos $(2,39)^{8}$. Las indicaciones señaladas en las puntuaciones medias de confianza se observan también en relación con un tema adicional ${ }^{9}$ : para la mitad de los encuestados, el sindicato lleva a cabo sus funciones de protección y promoción de los trabajadores de manera «poco satisfactoria». La propensión de los «satisfechos» para aparecer entre los favorables resulta especialmente marcada (el $65 \%$ frente a un promedio de «satisfechos» del 20,6\%).

\section{Una visión general}

El alto grado de participación y el consentimiento en el acuerdo firmado en julio de 2007 marca una visión única de «un importante acontecimiento de participación social» (Carrieri 2008: 10). Y es parte de una nueva tendencia de la democracia sindical. Según Baccaro (2007), el uso de estos procedimientos de consulta —el referéndum, y el de octubre de 2007- reforzará la capacidad de los sindicatos de participar en políticas centralizadas a través de dos tipos de mecanismos: agregativos y deliberativos. Expresado sintéticamente, la argumentación de Baccaro afirma que el mutado carácter de la negociación política, en particular la disminución de la disponibilidad de recursos para el intercambio, obliga al sindicato a invertir mucho más que en el pasado en procedimientos legitimizantes, como el referéndum sindical, en el que las decisiones de los vértices son objeto de debate y expresamente autorizadas por la base; se asume entonces un trade-off entre las contrapartes contractuales y los procedimientos democráticos: mientras más escasos sean las contrapartes (decommodifying) mayor es la inversión sindical en procedimientos democráticos de legitimación de las confederaciones sindicales. A diferencia del control jerárquico, la democracia sindical podría promover tanto la participación como la legitimidad; los individuos - incluso en ausencia de incentivos materiales - aceptan la elección colectiva convalidándola íntimamente (Baccaro 2008: 63).

Como en 1995, los sindicatos no han pedido simplemente a los trabajadores que expresen sus preferencias a través del voto: se promueven miles de reuniones en las que el contenido del acuerdo es cuidadosamente explicado y discutido con los trabajadores. Este estudio señala que los cambios de opinión al final son unos pocos (y cubren menos de un votante de cada diez); ha influido en la participación y en el voto más bien la cultura política y sindical, la actitud - la confianza — tanto en los sindicatos como en las instituciones en general. Por lo tanto, es una predisposición no «pun- 
tual» pero «temporal» (i.e. no a corto plazo). Se trata más bien de un renovado proceso de la democracia participativa, un espectro más amplio, político-social, ya puesto en marcha en 2005 con las primarias que promovieron Romano Prodi como candidato a premier de la alianza de centro-izquierda (La Unione); y en 2007, con las primarias del Partido Democrático que designaron la investidura como secretario de Veltroni ${ }^{10}$. Las estadísticas de participación en las elecciones primarias para la constitución del Partido Democrático señalan algunas tendencias que se observan incluso en el referéndum de los trabajadores (Venturino 2007): el PD resulta muy fuerte para atraer a los votantes del Sur, con puntas tal vez inesperadas en relación con la región de Campania (438 mil los votantes), Puglia (247 mil), Calabria (208 mil) y Sicilia (183 mil), y es fuerte también en las regiones con un antiguo modelo comunista (Toscana, Emilia, Umbria).

Considérese finalmente el último referéndum de los trabajadores de febrero 2009. En enero, los sindicatos Cisl y Uil firmaron un acuerdo con el gobierno Berlusconi por la reforma del sistema contractual; la Cgil no firma el acuerdo e invita igualmente a Cisl y a Uil para otra consulta (sobre el modelo de reforma de los contractos colectivos). A la vista de su negativa, la CGIL promueve la consulta por sí sola (la votación termina el 27 de marzo). En alrededor de cinco semanas la CGIL organiza 59.377 asambleas. Baste decir que en 2007 la CGIL, CISL y UIL organizaron 51.626 asambleas sobre el Protocolo sobre el Welfare. Por lo tanto, la única CGIL celebró 7.711 reuniones más. Votaron en las mesas electorales 3.643.836 trabajadores, jubilados, precarios. Por lo tanto, enfrentamos - todavía una vez más - un gran resultado en términos de participación y de voto: prueba de que la democracia sindical es un valor actual para los trabajadores, jubilados, precarios y que cada vez que se solicita un pronunciamiento la participación es muy alta porque la gente quiere determinar las decisiones que les incumben. El lema adoptado para la consulta es «Yo voto, yo decido».

Aunque dentro de un cuadro no-homogéneo (Meardi 2004), la acción sindical en Italia estaría complicada por varios factores, específicos al caso italiano (Cella 2003; Baglioni 2004): una parábola ascendente del sindicalismo y de la concertación (Carrieri 2007), fragmentación social y del mundo del trabajo, bajo nivel de confianza en las instituciones, menor disponibilidad de las expensas, desigualdad, pobreza (Istat 2008). A su vez, estos factores complican el sistema y las dinámicas de la representación social e sindical. Aunque el sistema social italiano esté marcado por estas características, el nivel de «pregunta de poder social» y de elección es muy fuerte, como se muestra en el caso de referéndum. Las formas de democracia participativa en Italia son varias y organizadas de forma bien diferente, pero, cuando el área sindical y política de la izquierda ofrece la posibilidad de escoger y decidir, la participación resta elevada (Baccaro 2004; Bobbio 2005; La Valle 2006; Cammelli 2005). Lo mínimo es poder elegir entre las opciones disponibles.

Este estudio no subraya un cambio en la cultura sindical pero sí un cuestionamiento social profundo de participación, deseo de elegir, que po- 
dría encontrar una respuesta política y sindical todavía más amplia: a la crisis institucional corresponde una demanda amplia de participación social. El dato más importante, sin embargo, es el de la participación y la democracia como poderoso medio de coordinación y equilibrio social en referencia a un amplio campo social, pero limitado. 


\section{Notas}

${ }^{1}$ La papeletas en blanco o nulas son 73.098 (1, 43\% del total): por lo tanto, es un valor contenido (si no insignificante).

${ }^{2}$ En otros casos, generalmente, la correlación entre las estadísticas socioeconómicas al nivel provincial (empleo, desempleo, las medidas de capital social) y la votación (participación y tipo de voto) muestran una relación de independencia o muy débil dependencia.

${ }^{3} \mathrm{Si}$ consideramos solamente los votantes, el porcentaje de «informados» se eleva al 68\%.

${ }^{4}$ Considere también que si entre los trabajadores los «informados o muy informados» son iguales a 51,7\%, entre los jubilados los informados suben hasta el 57,6\%.

${ }^{5}$ Y cabe recordar que en 2007, Romano Prodi es el Presidente del Consejo, apoyado por una coalición electoral de centro-izquierda.

${ }^{6}$ Se trata de un electorado que ya había adquirido firmes convicciones sobre la elección de voto; sólo el $11 \%$ de quienes afirman haber votado a favor del acuerdo ha cambiado idea en el período comprendido entre la firma del acuerdo y el referéndum en Octubre. Esta «rigidez» en el cambio de opinión se observa tanto entre los votos a favor que entre los que se oponen. Sólo el 7\% de los contrarios cambia de idea: en los meses entre julio y octubre de 2007, sólo el 7\% de los opuestos eran anteriormente favorables. Las opiniones sobre la bondad del acuerdo son con toda probabilidad ya adquiridas en el pasado (y también podrían ser entendidas como grado de acuerdo con las políticas del gobierno de Prodi). La razón de los pocos «cambios de opinión» no es atribuible a sólo uno y principal motivo, sino más bien a una más amplia gama de razones.

${ }^{7}$ La ANOVA comprueba si la diferencia entre los grupos (between) es significativamente mayor que la varianza dentro de los grupos (within) o se debe a las diferencias individuales distribuidos al azar en la población. Esta comparación se hace con la prueba estadística F de Fisher, que representa la relación entre la varianza-between y la varianza-within; por lo que cuanto más alto es el valor de F, mayor es la diferencia entre los promedios de los grupos. El análisis da apreciables valores de importancia para todos los ítems excluida la Iglesia Católica.

${ }^{8}$ Con marcadas diferencias entre los cuatro modos de tipología.

${ }^{9}$ Pregunta 46: En su opinión, o sobre la base de su experiencia, el sindicato italiano debe llevar a cabo sus funciones de protección y promoción de los trabajadores de manera: 1. «muy satisfactoria»; 2. «satisfactoria»; 3. «poco satisfactoria»; 4. «no es en absoluto satisfactoria».

${ }^{10}$ Así como, enseguida, en las elecciones primarias a Secretario del Partido Democrático de octubre de 2009, más de 3 millones de electores eligen a Pierluigi Bersani como Secretario del PD. 


\section{Bibliografía}

Accornero, A. (2007), “Cosa ci dice la consultazione”, Il diario del lavoro, 12, Octubre.

Altieri, G., Carrieri, M., Megale, A. (2007), "L’Italia del lavoro: un quadro d'insieme”, Quaderni di Rassegna sindacale. Lavori, a. VIII, n. 1.

Baccaro, L. (2004), "Stato e società civile: verso un modelo di democracia associativa?”, Stato e Mercato, 72.

Ídem (2007), Political economy della concertazione sociale, "Stato e mercato", n. 79.

Ídem (2008), "Contrattazione «politica» e consultazione dei lavoratori”, Quaderni di Rassegna sindacale, n. 1, IX.

Baglioni (2004), "Problemi e strategie dei sindacati di oggi", Stato e Mercato, 2004, 70.

Banca d'Italia (2008), “I bilanci delle famiglie italiane nell'anno 2006”, Supplementi al Bollettino Statistico, a. XVIII, n. 7, 28.

Bobbio, L. (2005), "La democrazia deliberativa nella pratica”, Stato e Mercato, 73, abril 2005.

Cammelli, M. (2005), "Considerazioni minime in tema di arene deliberative”, Stato e Mercato, 73 abril 2005.

Carbonai, D., Pedaci, M. (2009), "Il disagio economico tra i lavoratori subordinati. Risultati di un'indagine campionaria”, en Del Colle E. (2009), Disuguaglianze socioeconomiche e livelli di povertà, Franco Angeli, Milano.

Carrieri, M. (2008), L'altalena della concertazione. Patti e accordi italiani in prospettiva europea, Donzelli, Roma.

Cella, G. P. (2003), "La rappresentanza attraverso soggetti collettivi: rispecchiare o interpretare”, Stato e Mercato, 68, agosto 2003.

Feltrin, P. (2005), La sindacalizzazione in Italia (1986-2004), Edizioni Lavoro, Roma.

Istat, (2008), Rapporto annuale. La situazione del paese nel 2007, Istat, Roma.

La Valle, D. (2006), “La partecipazione alle associazioni in Italia. Tendenze generali e differenze regionali”, Stato e mercato, 77. 
Meardi, G. (2004), “Modelli e stili di sindacalismo in Europa?”, Stato e mercato, 71 .

Paci, M. (2007), “La famiglia e i sistemi di welfare nell’economia dei servizi”, en Regini, M., La sociologia economica contemporanea, Laterza, Bari.

Regalia, I. (2005), “Lavori atipici, nuovi, non-standard. Un campo ambivalente per l'analisi e l'azione sociale”, Sociologia del lavoro, n. 97

Ricolfi, L. (2005), “L’impoverimento dei ceti medi?”, Il Mulino, n. 1.

Venturino, F. (2007), “Le primarie nazionali dell’unione: un’analisi della partecipazione con dati aggregati”, Rivista italiana di Scienza politica, XXXVII, n. 3.

Recibido: 22.12.09

Aceptado: 16.06.10 